\title{
INFECTIOUS SPONDYLODISCITIS WITH PATHOLOGICAL FRACTURE PRESENTING AS PARAPARESIS: CASE REPORT
}

Bruno Sampaio Santos ${ }^{1}$, Joelma Moreira de Noroes Ramos ${ }^{1, \star}$, Cristiane Vieira Amaral ${ }^{1}$, Yane Chaves Martins Resende ${ }^{1}$, Tiberio Silva Borges dos Santos ${ }^{1}$, Jozelda Lemos Duarte ${ }^{1}$, Celso Soares Pereira Filho ${ }^{1}$, Cássia Gabrielle Alves Torres ${ }^{1}$, Vanessa Nepomuceno da Fonseca Meneses ${ }^{1}$, Laisa Allen Gomes de Sousa ${ }^{1}$

1.Universidade Estadual do Piauí, Teresina (PI), Brazil.

*Corresponding author: joelmanoroes@hotmail.com

\section{BACKGROUND}

Spondylodiscitis is characterized by an infectious process of the intervertebral disc with the possibility of affecting adjacent areas, which can evolve with compression of nervous structures and determine neurological symptoms. The main route of transmission is hematogenous and is commonly associated with trauma, intravenous substances, hemodialysis and immunosuppression. The aim of this report is to present an advanced neurological condition of a patient with multiple comorbidities who was using corticosteroid at an immunosuppressive dose for chronic pain.

\section{CASE REPORT}

MSM, female, 60 years old, divorced, housewife, with hypertension and diabetes, former smoker, reported progressive paraparesis of the lower limbs with preserved sensitivity for 1 year, associated with moderate back pain, which made it difficult to perform daily tasks, showing partial improvement with the use of anti-inflammatory drugs and corticoids. She reported weight loss of $10 \mathrm{~kg}$ in this time interval and denied fever. One month before admission, the patient suffered a fall from a height of $1 \mathrm{~m}$ and evolved with paraplegia and urinary retention. In the initial tests, normocytic and normochromic anemia, leukocytosis with a left shift, elevated CRP and ESR, negative blood cultures and normal renal function were observed. Chest tomography showing lytic lesion in the thoracic vertebra, with compressive fractures of the vertebral bodies of T6, T7 and T8 and spine resonance showing perivertebral collections, determining medullary compression, suggestive of spondylodiscitis, with broad-spectrum antibiotic therapy being instituted. Bone scintigraphy with radiopharmaceutical uptake in the thoracic spine and costal arches. Normal total abdomen tomography; protein electrophoresis without monoclonal peak. Complementary investigation performed: negative tumor markers, unaltered breast and thyroid ultrasound, nonreactive PPD. The perivertebral abscess was drained, and the material was sent for cultures and histopathological analysis, which showed a negative result for neoplasm. The patient evolved with clinical and laboratory improvement, maintaining the neurological status. She was discharged for outpatient follow-up.

\section{CONCLUSION}

Intervertebral disc infection can have an insidious course and, in many cases, it can present with severe manifestations. Thus, among the various causes of spondylodiscitis, prolonged use of corticosteroids may have influenced the severity of the condition, causing immunosuppression and the possibility of osteoporotic fractures.

\section{KEYWORDS}

Spondylodiscitis, Chronic, Low back pain. 